\title{
Atomic-Scale Lightning Rod Effect in Plasmonic Picocavities: a Classical View to a Quantum Effect
}

\author{
Mattin Urbieta, ${ }^{\dagger}+$ Marc Barbry, ${ }^{\dagger}$ Yao $^{\prime}$ Zhang, $^{\dagger}$ Peter Koval $^{\dagger}{ }^{\dagger}$ Daniel \\ Sánchez-Portal, ${ }^{\dagger}$ Nerea Zabala, ${ }^{*}, \dagger, \uparrow$ and Javier Aizpurua*, ${ }^{*}$ \\ Materials Physics Center (CSIC-UPV/EHU), and Donostia International Physics Center \\ (DIPC), Paseo Manuel de Lardizabal 5, 20018 San Sebastián, Spain, and Department of \\ Electricity and Electronics, FCT-ZTF, UPV-EHU, 48080 Bilbao, Spain \\ E-mail: nerea.zabala@ehu.eus; aizpurua@ehu.eus
}

\begin{abstract}
Plasmonic gaps are known to produce nanoscale localization and enhancement of optical fields, providing small effective mode volumes of about a few hundreds of $\mathrm{nm}^{3}$. Atomistic quantum calculations based on Time-Dependent Density Functional Theory (TDDFT) reveal the effect of subnanometric localization of electromagnetic fields due to the presence of atomicscale features at the interfaces of plasmonic gaps. Using a classical model, we explain this effect as a non-resonant lightning rod effect at the atomic scale that produces an extra enhancement over that of the plasmonic background. The near-field distribution of atomic-scale hot spots around atomic features is robust against dynamical screening and spill-out effects, and follows the potential landscape determined by the electron density around the atomic sites. A detailed comparison of the field distribution around atomic hot spots from full quantum atomistic calculations and from the local classical approach considering the geometrical profile of the atoms electronic density, validates the use of a classical framework to determine the field distribution and effective mode volume in these extreme subnanometric optical cavities. This finding is of practical importance for the community of surface-enhanced molecular spectroscopy and quantum nanophotonics, as it provides an adequate description of the local electromagnetic fields around atomic-scale features with use of simplified classical methods.
\end{abstract}

\section{Keywords}

nanoplasmonics, picocavities, lightning rod effect, ab initio calculations, effective mode volume

Progress in nanotechnology has allowed to control the morphology of metallic nanoparticles at the nanometer and even sub-nanometer scale, ${ }^{1-5}$ allowing for development of various applications in plasmonics and nanooptics, such as in enhanced vibrational spectroscopy, ${ }^{6-10}$ improvement of energy absorption of solar cells, ${ }^{11,12}$ optoelectronic circuits, ${ }^{13}$ quantum optics, ${ }^{14-16}$ nanosensing of biomolecules, ${ }^{17,18}$ or non-invasive thermo-therapy in medicine. ${ }^{19}$ Most of the fascinating properties and applications of plasmonic nanoparticles are based on the tunability of their optical response, along with their ability to enhance electromagnetic fields squeezing the electromagnetic energy down to nanometer scale volumes around sharp tips or at interparticle gaps producing "hot-spots", behaving as effective optical nanoantennas. ${ }^{20-23}$ This is possible through the excitation of localized surface plasmons that couple efficiently to light, allowing to overcome the diffraction limit. ${ }^{24}$ The near-field patterns in nanostructures under light excitation strongly depend on the size, composition and 
shape of the individual particles, along with plasmon hybridization in coupled nanostructures.

Light scattering of nanoparticles of arbitrary shape and size is usually well addressed within a classical electrodynamics framework (with the use of a suitable dielectric function), by solving Maxwell's equations for specific compositions, morphologies and environments. ${ }^{25-35}$ When the size or the separation distance between plasmonic nanoparticles becomes of the order of a few nanometers or even smaller, the quantum nature of the electrons emerges due, among others, to the particle-size effect in the electron confinement, ${ }^{36-40}$ the inhomogeneous dynamical screening of the electrons response, ${ }^{41,42}$ the electron-spill out at the metal interfaces, ${ }^{43}$ the presence of atomistic inhomogeneities, ${ }^{44,45}$ or even the activation of quantum tunneling ${ }^{46,47}$ across subnanometer interparticle gaps. All of these effects are initially not included in typical local classical electrodynamical descriptions of the optical response, and different levels of approximation have been adopted to address their influence in the response in extended classical models. ${ }^{48-51}$ Among all these effects, the presence of atomic-scale features at the surfaces has not been deeply explored in the context of plasmonics up to recently, due to the intrinsic limitations of most of the phenomenological classical models which do not address the quantum effects mentioned above. A proper description of the effect of atomic-scale edges, wedges, vertices, and protrusions at surfaces requires a complete quantum theoretical framework which includes the atomistic structure of the nanoparticles and the wave nature of electrons building up the plasmonic excitations.

$A b$ initio atomistic methods provide an appropriate quantum framework to consider the aforementioned effects including the atomistic structure in a straightforward and complete manner, ${ }^{45,52-55}$ with the drawback of being computationally demanding. Recently, a few works ${ }^{44,45,56,57}$ have studied the impact of the atomistic structure on the optical response of metal clusters of a few nanometers and dimers within atomistic timedependent density functional theory (TDDFT) ${ }^{58,59} \mathrm{cal}-$ culations, showing that the atomistic structure at the interfaces of a metallic nanostructure need to be considered for an accurate description of the local field distributions around atomic-scale features. It has been shown ${ }^{45}$ that the presence of single atoms, or atomic edges in facets of the nanoparticles allows to localize and confine the near-field down to subnanometric dimensions, well below the underlying plasmonic background. This has enabled a possible route towards photonics at the picoscale, where the localization of local fields in atomic-scale cavities leads to extremely small effective mode volumes, thus boosting the coupling of photons with the electronic transitions of single emitters, ${ }^{60}$ or with the vibrations of a molecule in optomechanical interactions. ${ }^{61}$

The quantum description of these optical picocavities at the full atomistic level reveals the importance of atomic-scale features. However, such detailed descrip- tion is often limited by the computational requirements, even with the relatively efficient TDDFT methods. Here we propose the use of a simplified local classical approach to address the optical response and the local field distribution around picocavities. By adopting a sharp boundary interface that coincides with the profile of the atomistic distribution within a plasmonic structure we show that a faithful reproduction of the near-field properties of the picocavity can be achieved. The proposed methodology is capable to expose the extreme nanophotonic properties of these picocavities embedded in larger structures, allowing standard methods of electrodynamics to address this challenging regime. ${ }^{62}$ The concept proposed here thus impacts nanoscale condensed matter physics, quantum chemistry, and nanophotonics equally.

\section{Results and discussion}

In order to address how faithfully a method based on classical electrodynamics can approach an atomistic, quantum mechanical description of the optical response and the field distribution around atomic-scale features in plasmonic structures, we shall compare the results of the calculations obtained within each of the methodologies. We analyse the optical response (absorption cross section), near-field enhancement, and localization in a single sodium cluster as well as those in the gap between two sodium clusters. Our focus is placed on the distribution of field enhancement around the particles strong geometrical inhomogeneities. Na clusters are used as examples of metallic nanoparticles, however our conclusions can be extended to other materials and geometries when considering the appropriate parameters (dielectric function, basic geometry and atomic radius). The quantum and classical approaches used here are described in the Methods section, as well as in the SI.

\section{Field localization in atomic-scale morphologies}

First we explore the optical response and field localization around atomic-scale features in isolated particles. To that end we perform calculations of the absorption cross section and near-field distribution in single $\mathrm{Na}$ icosahedral clusters. The quantum atomistic calculations adopt the geometry shown in Figure 1(a), whereas the classical local calculations adopt the smooth icosahedral shape displayed in Figure 1(b). Additionally, the situation of a smooth spherical geometry with no atomic-scale features is also considered. The absorption cross section obtained from the three models is compared in Figure 1(c). In the quantum calculation (blue line) we observe a single peak at $3.15 \mathrm{eV}$, corresponding to the dipolar plasmon (DP) resonance, and a shoulder at around $3.8 \mathrm{eV}$. Notice a red-shift of about $0.2 \mathrm{eV}$ of the DP with respect to the results reported in Ref. ${ }^{45}$, due to a more adequate material's density 
a)

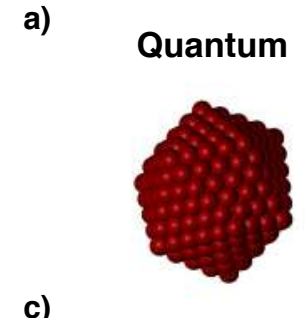

c)

b)

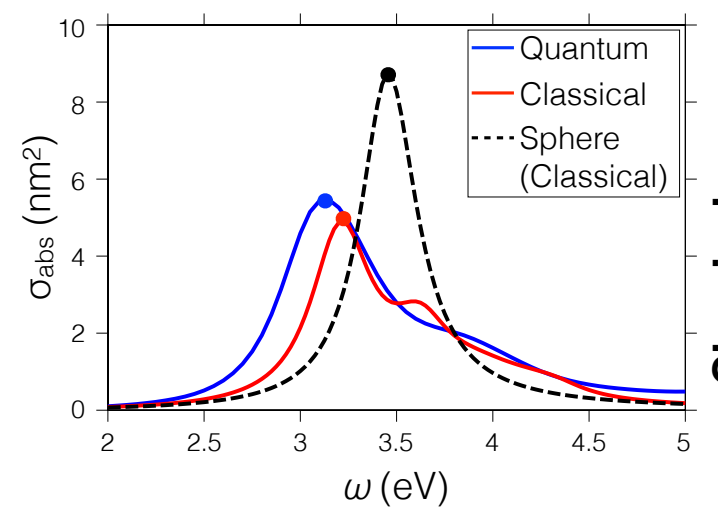

Classical
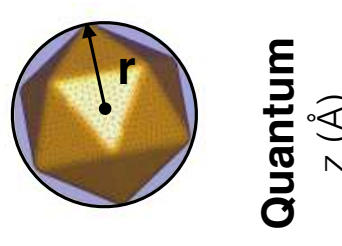
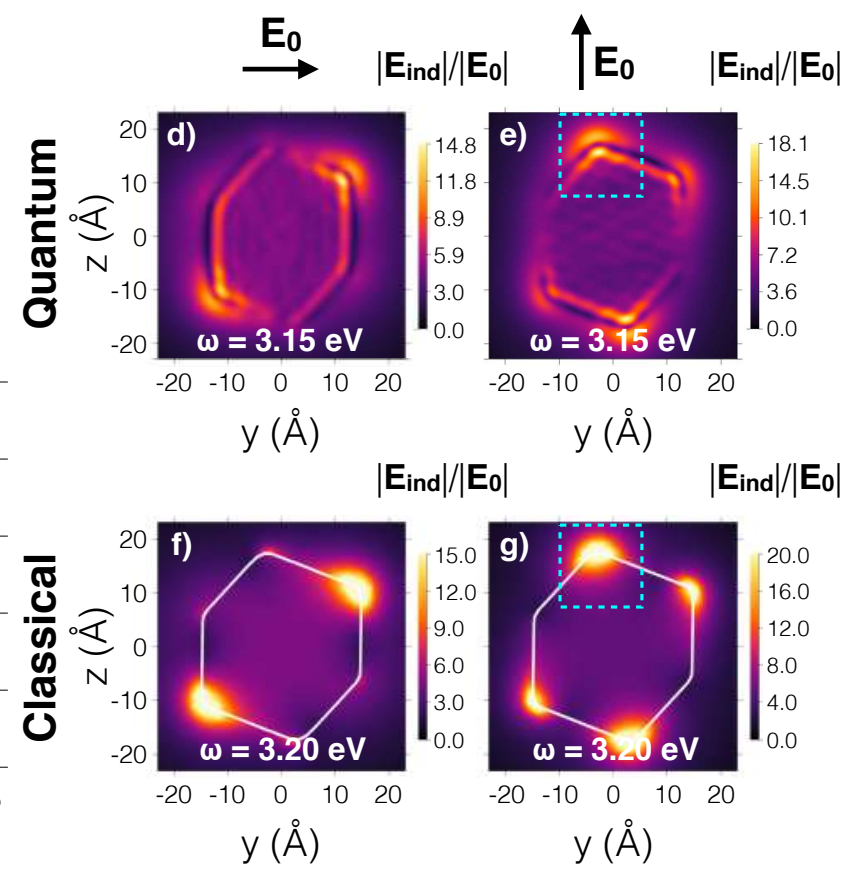

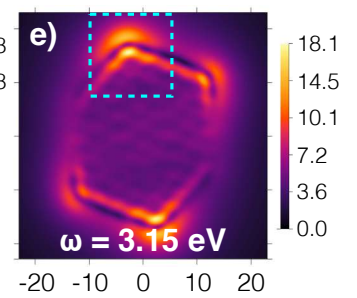

1020

y $(\AA)$

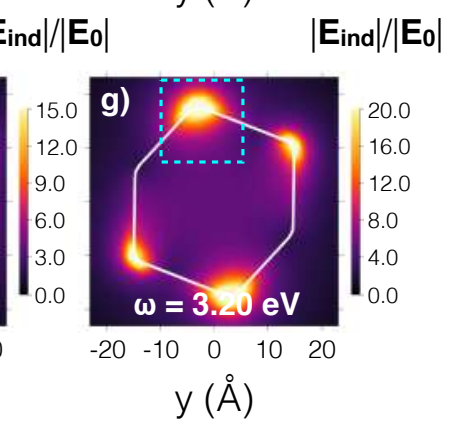

Figure 1: (a) Atomistic cluster composed by 380 sodium atoms used in TDDFT calculations. (b) Sketch of the continuous icosahedral cluster used for BEM calculations. The sphere of radius $r=18.5 \AA$ containing the icosahedron has been drawn for reference. The tips and edges have been rounded to have a minimum radius of $r_{\mathrm{Na}}=2.08 \AA$. (c) Absorption cross-section of the particle calculated using TDDFT (blue line) and BEM (red line). The energies of the dipolar plasmons obtained for both models are marked by round dots. The dashed black line corresponds to the absorption cross section of the circumscribing sphere in $b$ ), as calculated with BEM. A peak corresponding to a higher order plasmon (HOP) is observed for BEM at $\omega=3.6 \mathrm{eV}$, while a shoulder is appreciated for TDDFT around $\omega \sim 3.8 \mathrm{eV}$. (d)-(g) Induced near-field enhancement for a single icosahedral nanoparticle obtained using TDDFT (d),(e) and BEM (f),(g). The external incident field amplitude, $\mathrm{E}_{0}$, is polarized along the $y$ axis (left column) and along $z$ axis (right column).

provided by the GGA relaxation. In the classical calculation (red line), two clear peaks emerge at $3.20 \mathrm{eV}$ and $3.6 \mathrm{eV}$, corresponding to the $\mathrm{DP}$ and a higher-order plasmon (HOP) modes, respectively. The difference in the energies of the DP mode in both models is minimal, but more pronounced differences can be found in their intensity. Moreover, when smoother geometries are considered in the classical BEM calculations, a dominance of the DP mode and a smearing of the HOP mode are observed (see SI for further details). This explains the presence of a clear second peak in the classical sharp description, compared to the shoulder obtained in the full quantum calculation, rounded by the electron cloud spilling at the interfaces. The spectrum corresponding to a classical spherical particle, of size as sketched in Figure 1(b) is also plotted (dashed black line) for reference. A single peak corresponding to the DP resonance is observed at $3.45 \mathrm{eV}$ in the smooth spherical particle. The difference in the intensity of the DP arises in part due to the presence of higher order modes in the case of the icosahedral particle.

One of the most important aspects of an optical resonator is its capacity to localize its optical modes to the smallest effective volume as possible. In Figures $1(\mathrm{~d}-\mathrm{g})$ the field distribution around the Na nanoparticle is shown for two perpendicular polarizations of the in- cident electromagentic field. Results for the fully quantum atomistic approach corresponding to the structure of $380 \mathrm{Na}$ atoms, stable within the DFT energy landscape [Figures $1(\mathrm{~d}, \mathrm{e})]$, are compared with those obtained within a local classical approach that adopts an icosahedral structure that approximately follows the electron density profile of the atomistic calculation. The tips and edges are smoothened in such a way that the maximum curvature of their metal-vacuum surfaces correspond to the Wigner-Seitz radius of sodium $\left(r_{s}=2.08 \AA\right)$. All the plots correspond to the energy of the DP resonance. Data are shown in the $(y, z)$ plane passing through the center of the particle. The polarization of the incident field is parallel to the $y$-axis for the plots on the left (d,f), and along the $z$-axis for the plots on the right $(\mathrm{e}, \mathrm{g})$. The atomistic structure is clearly appreciated in the TDDFT results (top row), and as one might expect, there is no atomistic contrast in the classical near-field maps (bottom row), due to the continuous description of the media interfaces within this model. Remarkably, both models describe very similarly the effect of localization of the near field at the atomic protrusions within the particles interfaces (at the tips and edges of the particles), giving rise to pronounced atomic scale "hot spots". These subnanometric-scale hot spots are clearly identified in all the field distributions on top 
of the plasmonic nanometric background, and they are also correctly captured by the classical local description. One can thus conclude that it is possible to describe the main features of subnanometric localization of the fields if the atomistic structure of the particle is correctly approximated by a smooth and continuous surface. These results set the validity of classical approaches to address the actual local field distribution around atomistic features, even in the most extreme situations of localization. With this result at hand, it can be proposed that atomic-scale hot spots in large plasmonic configurations can be correctly tackled by a proper classical approach which accounts for the geometrical profile of the atomistic structure. We analyse deeper now the physical mechanism underlying this extreme atomic-scale optical localization.

\section{Atomic-scale lightning rod effect}

The extra-localization obtained around the atomistic features of a smooth metallic body is sustained by a non-resonant effect that is superimposed on the overall plasmonic enhancement produced by the collective oscillation of the electronic surface charge density in the nanoparticle. At the macroscopic scale, it is well known that regions which expel the electric field and present a pronounced geometrical curvature, produce an abrupt change of the electrical potential, $\phi(\mathbf{r})$, in their proximity (large potential gradient), ${ }^{63}$ and thus strong induced electric field, $E_{\text {ind }}$, since $E_{\text {ind }}=-\nabla \phi(\mathbf{r})$. The field enhancement following a strong potential gradient due to the curvature of a metallic interface is commonly described as the "lightning rod effect". In analogy to the macroscopic situation, in the proximity of an atomic protrusion, an atomic-scale lightning rod effect is produced which is responsible for the extra localization at the tips and edges of an interface [see sketches in Figures 2(a)]. In spite of the strong quantum effects that govern the optical response of the electron gas at the subnanometric scale, including dynamical screening and spill-out, the main features of the field localization and enhancement in atomic protrusions can be correctly addressed by the consideration of the sharp curvature of the atomistic profile, as demonstrated with the quantum and classical calculation of this effect in Figures $1(\mathrm{~d}-\mathrm{g})$. A zoom-in of the near-field around one of these protrusions, marked with blue dashed squares in Figures $1(\mathrm{e}, \mathrm{g})$, is displayed in more detail in Figures $2(\mathrm{~b}, \mathrm{c})$. The classical calculation faithfully reproduces the spatial extension and the intensity of the quantum atomic-scale localization, in accordance with a field distribution that corresponds to a protrusion with the size of the atomic radius.

A quantitative estimation of the atomic-scale lightning rod effect can be inferred from a quasistatic analysis of the field distribution around a semispheroidal feature on top of metallic flat interface [see schematics in Figure 2(d)]. The exemplary semispheroidal feature mimics an atomic protrusion and is characterized by a semiwidth $b$, and a semiheight $a$, as depicted in Figure $2(\mathrm{f})$. We consider a background plasmonic field $E_{\mathrm{bp}}$, which would be the induced field in a spherical particle without corrugations, and calculate the induced field $E_{\text {ind }}$ distribution and intensity around the semispheroidal protrusion for two distinctive situations: (i) a featureless protrusion of very small height $(a / b \approx 0.1)$ in Figure 2(e), and (ii) half an atom protruding from the flat surface $(a / b \approx 1)$ in Figure $2(\mathrm{f})$, mimicking the supporting particle surface (of much larger radius). Whereas the featureless protrusion hardly provides any field enhancement $\left(\left|E_{\text {ind }}\right| /\left|E_{\mathrm{bp}}\right| \approx 1\right)$, the atomic-sized protrusion produces a three-fold extra enhancement over the incident background field. The full set of field enhancements obtained for all the different values of $a / b$ are shown in Fig 2(g), which show the quasistatic enhancement of the induced field with respect to the background field, $\left|E_{\text {ind }}\right| /\left|E_{\mathrm{bp}}\right|$ as a function of the dimensions of the atomic-scale protrusion ( $a$ and $b$ ). The simple expression of this enhancement is given in the inset of Fig $2(\mathrm{~g})$ (see derivation of this expression in SI). This simple estimation is in very good agreement with the actual values obtained from the classical and quantum calculations (blue and green dots in Figure $2(\mathrm{~g})$, respectively), validating the interpretation of a non-resonant lighting rod effect at the atomic scale as responsible for the extra enhancement observed in atomistic protrusions.

\section{Atomistic plasmonic nanogaps}

As pointed out in the introduction, one of the best canonical plasmonic resonators is given by the formation of a metal-insulator-metal (MIM) structure, connecting two metallic nanostructures at a nanometric separation distance, forming a plasmonic nanogap. In order to explore the properties of local field enhancements and effective mode volumes in plasmonic nanocavities, we consider three different configurations of metallic dimers formed by the sodium clusters analysed in the previous section, according to their mutual orientation. The icosahedral clusters present atomistic features and are aligned across the gap in configurations referred to as (i) facet-to-facet, (ii) tip-to-facet and (iii) tip-to-tip, as sketched on the top panels of Figure 3. Moreover, the separation distance also affects the properties of the system and will be considered as a parameter.

Before analysing the local field and the effective mode volume, it is useful to address the far-field response for each plasmonic gap configuration as a function of interparticle distance, and compare thr results obtained within quantum TDDFT and classical BEM descriptions. We perform calculations of the absorption cross sections for the three different gap geometries, sketched in the upper row of Figure 3, corresponding to different particle orientations, namely facet-to-facet (a), tip-tofacet (b) and tip-to-tip (c). We trace the optical modes of the cavities from a separation distance of $d_{\mathrm{sep}}=20 \AA$, to a situation of touching and overlapping of the parti- 
a)

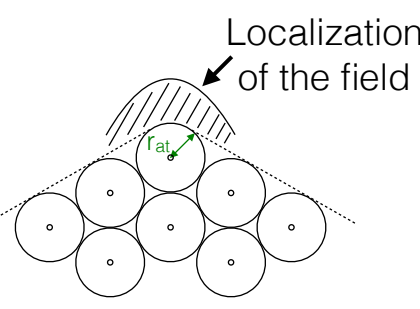

b)

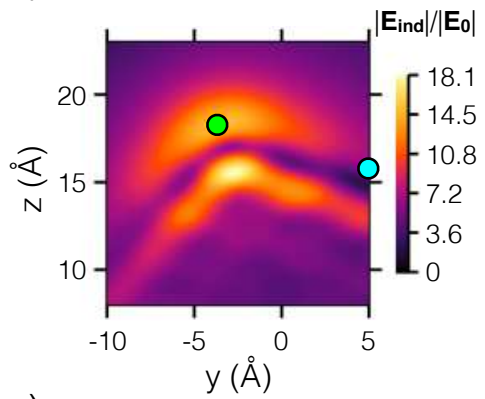

c)

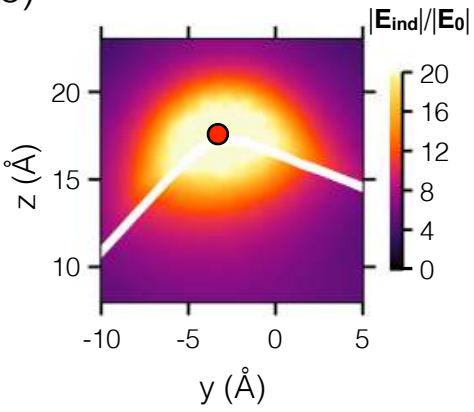

d)

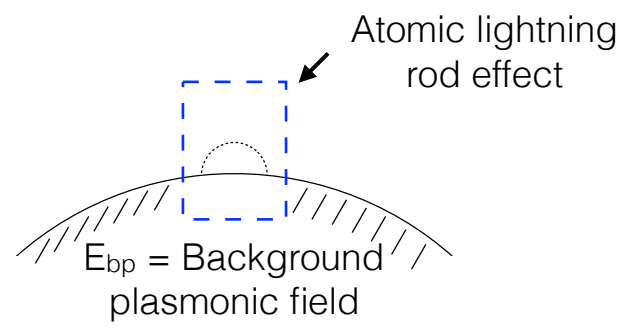

e)

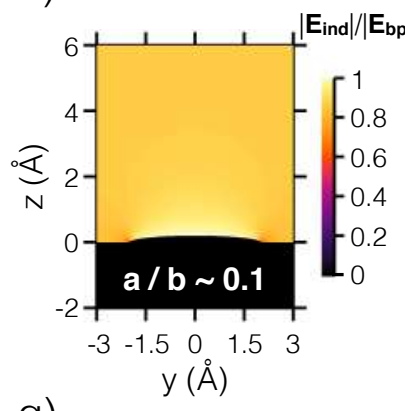

f)

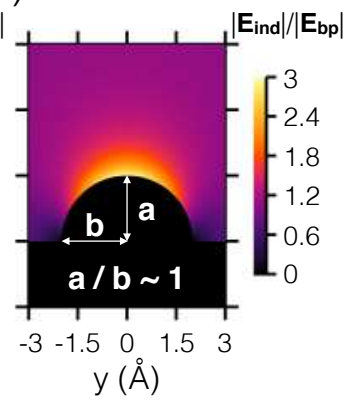

g)

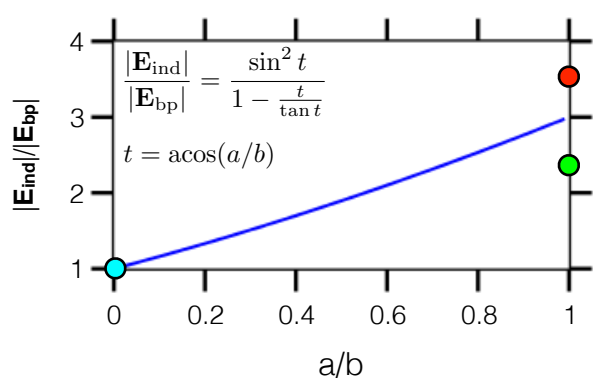

Figure 2: (a) Sketch showing the tip corresponding to the icosahedral atomistic nanoparticle used in TDDFT (circles represent atoms). The dotted line along the nanoparticle interface shows the surface used in the BEM calculations. The zone where the field is localized has been highlighted with oblique lines. (b) Zoom-in of the region enclosed by the cyan dashed lines of the TDDFT near-field maps from Figure 1(e), and (c) zoom-in of the BEM near-field maps from Figure $1(\mathrm{~g})$. (d) Sketch of an atomic protrusion on a spherical nanoparticle that produces an atomic lightning rod effect enhancing the background plasmonic field given by the spherical nanoparticle. (e) Near-field enhancement map of the induced electric field $\left|E_{\text {ind }}\right|$ with respect to the background plasmonic field $\left|E_{\mathrm{bp}}\right|$, corresponding to an almost flat protrusion $(a / b \sim 0.1)$, with practically no enhancement of the field. (f) Near-field map enhancement as in (e), corresponding to a spherical protrusion $(a / b \sim 1)$, giving a maximum field enhancement, $\left|E_{\text {ind }}\right| /\left|E_{\mathrm{bp}}\right|$, of $\approx 3$. (g) Field enhancement at the boundary $(x=0, z=a)$ of an oblate spheroidal protrusion as a function of the aspect ratio. The colored dots correspond to the field enhancements at the positions marked in panels (b) and (c). The enhancement of the induced fields has been calculated with respect to the plasmonic background field, $\left|E_{\mathrm{bp}}\right|$, obtained at the boundary of a typical spherical surface, which is of the order of $\left|E_{\mathrm{bp}}\right| \approx 6$.

cles $\left(d_{\text {sep }} \leq 4 \AA\right)$. For the atomistic approach, within TDDFT, the separation distance has been set as the distance between closest atoms from opposite clusters $d_{\text {sep }}=d_{\text {at }}$ (see SI for further details). In the case of the continuous classical description, the separation distance is considered as the distance between the closest points of the particles surfaces $d_{\mathrm{c}}$, which includes an additional correction term corresponding to the distance between the center of an outermost sodium atom and the surface of the particle (the $\mathrm{Na}$ atoms have been considered as spheres of radius $\left.r_{\mathrm{Na}}=2.08 \AA\right), d_{\mathrm{sep}}=d_{\mathrm{c}}+2 \cdot r_{\mathrm{Na}}$. The modeling of the overlapping situation within each approach is further explained in the SI (see Figure S2).
No further relaxation has been considered for the case of merging particles. An alternative approach consisting in removing subsequent layers of overlapping layers of atoms in the junction has been adopted. The polarization of the incident light is oriented along the dimer axis.

The absorption cross section of all the plasmonic gaps is mainly characterised by the existence of a bonding dimer plasmon (BDP) that slightly redshifts when the two particles approach. ${ }^{64,65}$ This effect is very clear in the classical calculations (bottom row in Figure 3) and appears as less pronounced in the quantum results. For large separation distances $\left(d_{\text {sep }}>6 \AA\right)$ the gap modes 
Facet to facet

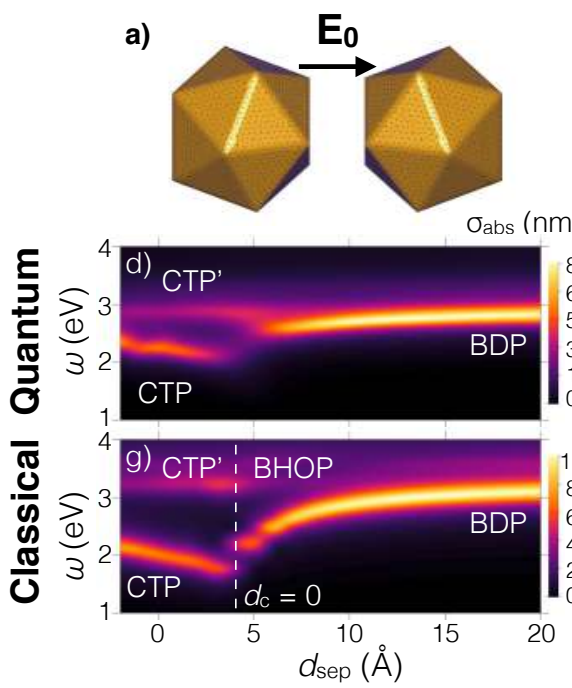

a)
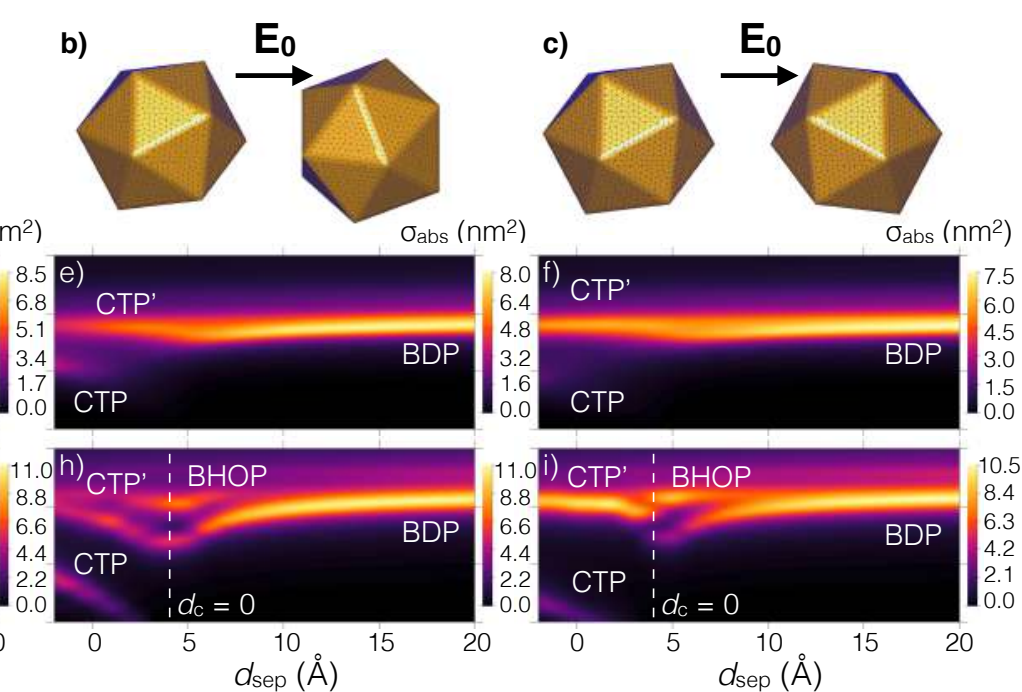

Figure 3: Sketch of plasmonic dimers with the three interparticle gap configurations considered, (a) facet-to-facet, (b) tip-to-facet and (c) tip-to-tip. The polarization of the incident electric field, $\mathrm{E}_{0}$, is always oriented along the dimer axis. ( $d-f$ ) Absorption cross-section spectra of the corresponding icosahedral dimers displayed on top, as a function of the interparticle separation distance $d_{\mathrm{sep}}$, as obtained with the use of the TDDFT. (g-i) Same as in (d-f), obtained with the use of classical BEM calculations. The hybridized bonding dimer plasmon (BDP) mode, bonding higher-order plasmon (BHOP) mode, charge transfer plasmon (CTP) mode and higher-order charge transfer plasmon (CTP') mode are indicated in the spectra for those cases where such identification is possible. The minimum distance $\left(d_{\mathrm{c}}=0 \AA\right)$ for which the continuous (BEM) particles overlap is marked with dashed white lines.

appear at a similar energy for all the configurations considered.

In the atomistic TDDFT calculations (second row in Figures 3(d-f) the BDP slightly redshifts as the interparticle distance decreases, and for a separation smaller than $6 \AA$ it eventually fades away, indicating that the quantum tunneling regime has been reached. ${ }^{45}$ At those separation distances, the charge transfer plasmon (CTP) at lower energy (around $2 \mathrm{eV}$ ) emerges due to the tunneling current, even if the particles are not in physical contact $\left(d_{\text {sep }} \sim 5 \AA\right.$, while the interatomic distance is $\sim 4 \AA$ ). Notice the higher intensity of the CTP mode for the facet-to-facet geometry with respect to the other two configurations, due to a larger particle surface area exposed at the minimum gap distance, which includes a greater overlapping of the electron densities at the clusters gap interfaces. Furthermore, for these short distances the higher-order charge transfer plasmon (CTP') mode is also excited at higher energy (about $3 \mathrm{eV}$ ). Notice that for tip-to-facet and tip-totip geometries in Figures 3(e-f), the CTP' modes have larger relative weight than the lowest order CTP mode, as the tunneling transport of the electrons through the gap is reduced when going through a tip, rather than in a situation of facet-to-facet tunneling [Figure 3(d)].

Similarly to the situation of the isolated nanoparticle, we also mimic the configuration of the plasmonic gaps of different morphologies with use of a classical approach based on the BEM. Overall, the optical response cal- culated within the classical approach is similar to the quantum one for $d_{\text {sep }}>6 \AA$. For this classical separation range, a larger redshift of the BDP mode can be observed in the classical description compared to what is obtained in the TDDFT calculation. This behavior is also observed for dimers composed by spheres, ${ }^{64}$ and it is enhanced here by the extreme geometrical features of our system. This can be clearly ilustrated by comparing the facet-to-facet gap $(\mathrm{g})$ which shows a slight excitation of the bonding higher order plasmon (BHOP) and an intense BDP mode, and the tip-to-tip (i) with a much stronger BHOP excitation and lower BDP presence.

Beyond this classical regime, major differences with respect to the TDDFT results are observed in the $2 \AA<$ $d_{\text {sep }}<6 \AA$ separation range, where electron spilling effects and a strong tunneling affect the optical spectra. A local classical electrodynamical approach cannot reproduce these effects due to their pure quantum nature, even though novel effective approaches have been developed to account for them within extended classical treatments. ${ }^{48}$

Finally, for overlapping particles with $d_{\text {sep }}<0 \AA$, the pattern observed in the classical spectra, characterized by a distribution of charge transfer plasmons (CTPs and CTP's), reproduces that of the TDDFT results, although the relative intensities of CTP and CTP' are dramatically dependent on the geometry and width of the neck connecting the particles. As far as the optical spectrum is concerned, most of the spectral features are 
well reproduced by the classical approach, identifying clearly the bonding plasmon at the gap and the charge transfer when a spatial overlap is small. The tunneling regime for separations below $6 \AA$ requires further elaboration, as shown in the literature. ${ }^{48}$

\section{Extreme effective mode volumes: picocavities}

The gap plasmon identified for the three morphologies in Figure 3 is a canonical mode in plasmonics widely exploited as a building block for molecular sensing and spectroscopy. ${ }^{66-69}$ We now analyse the validity of the classical approach to describe the local field distribution and the effective mode volume of the gap mode. We focus on the face-to-tip configuration and analyse the local field distribution for different separation distances within both quantum and classical approaches. We select this configuration because it gathers both tip and facet features and thus illustrates adequately the outcome for each morphology. In Figure 4, we show the induced field for four different particle separations, namely $d_{\text {sep }}=20 \AA$ (first row, a,b), $d_{\text {sep }}=10 \AA$ (second row, c,d), $d_{\text {sep }}=6 \AA$ (third row, e,f) and $d_{\text {sep }}=1 \AA$ (fourth row, g,h), have been considered to illustrate the different interaction regimes: weak interaction, medium interaction, strong interaction and charge transfer regimes.

Quantum results are displayed in the left column of Figure 4 and classical results in the right column. In the weak interaction regime, $\left(d_{\text {sep }}=20 \AA\right.$, a,b) the nearfield distribution resembles that in isolated single particles. Nevertheless, a larger enhancement of the field is observed in the gap, meaning that an hybridization of the dipolar modes of the individual particles into the BDP mode also emerges at this separation distance. Classical results reproduce the presence of the atomicscale hot spots not only in field distribution but also in the intensity of the enhancement produced (of the order of 25 fold). As the nanoparticles gap is decreased the BDP gets more localized in the gap [Figures $4(\mathrm{c}, \mathrm{d})$ ]. Both approaches predict the progressive localization of the medium and strong interaction regimes, with values of the field enhancement at the gaps of around 35 fold. Finally, as the nanoparticles overlap [Figures $4(\mathrm{~g}, \mathrm{~h})$ ], charge transfer across the particles is produced, expelling the field from the gap, an effect correctly captured by the classical approach. As observed in the comparison between quantum and classical results in Figure 4, the classical approach provides a very adequate framework to address the local-field distribution around atomic-scale features, even in the extreme siuation of small clusters where quantum effects are more pronounced. We can thus conclude that this classical approach can be safely used to describe atomic-scale features in much larger plasmonic structures, which cannot be directly tackled by quantum methods.

The near-field maps in the charge transfer regime, plotted for the CTP' mode energies in Figures $4(\mathrm{~g}, \mathrm{~h})$ reveal that this regime can be also correctly captured by means of a classical description. In the case of the TDDFT description (panel g) the charge is transferred through the "gap" due to the overlap of the electron wavefunctions, so that the field is expelled to the surrounding of the gap and the field confinement decreases. The same occurs in the classical BEM description (panel h), where the particles profile follow the overlapping neck of the particles electron density, and thus the field is also expelled to the outside with a very similar pattern to the TDDFT result. Moreover, the main features of the near-field distribution around the tips are also preserved in both models.

One of the most important characteristics of an optical resonator is the effective mode volume associated to the resonance modes sustained by the resonator. When an atomic-scale lightning rod effect is mounted on the field of a plasmonic resonance, a slight modification of the field-enhancement and the effective mode volume is produced by the picocavity. As we have illustrated throughout this work, the classical description of the atomic-scale features turns to be a very adequate framework to address the properties of these picocavities. We thus compare now the maximal field enhancement and effective mode volume obtained in the different plasmonic cavities as a function of gap separation distance, $d_{\text {sep. }}$. Classical and quantum results for the effective mode volumes are gathered in Figure 5, for three mutual orientations considered earlier in this work. Maximum near-field enhancement at the center of the gap $\left|\mathbf{E}_{\text {ind }}^{\max }\right| / \mathbf{E}_{0}$ is shown in panels (a-c) of Figure 5, and the corresponding effective mode volume $V_{\text {eff }}$ in panels (df). For separation distances larger than $d_{\text {sep }} \sim 8-10 \AA$, the maximum induced near-fields show similar trends for all the configurations with excellent agreement between the quantum and the classical descriptions. For smaller separation distances, the quantum model accounts for the quenching of the local field enhancement produced by the quantum tunneling at optical frequencies, ${ }^{70}$ whereas the classical picture provides an unphysical increase of the local field. The quantum description (TDDFT) addresses the existence of a maximum of local field at $d_{\text {sep }}=8 \AA$ for the facet-to-facet configuration, which is slightly shifted to a smaller separation for the other configurations showing atomic-scale protrusions ( $d_{\text {sep }}=7 \AA$ for tip-to-facet and tip-to-tip configurations), due to a reduced effect of the tunneling in those configurations. In the classical description, the enhancement of the local fields increases exponentially as the gap is closed, with larger values for tip-to-tip and tip-to-facet configurations due to a more pronounced lightning rod effect. In light of these results one can conclude that the classical description of the atomic scale local fields is correct until separation distances of about $8 \AA$.

Along with the maximum absolute value of the field enhancement, the corresponding effective mode volume, $V_{\text {eff }}$ for each situation is shown in Figures $5(\mathrm{~d}-\mathrm{f})$. This 

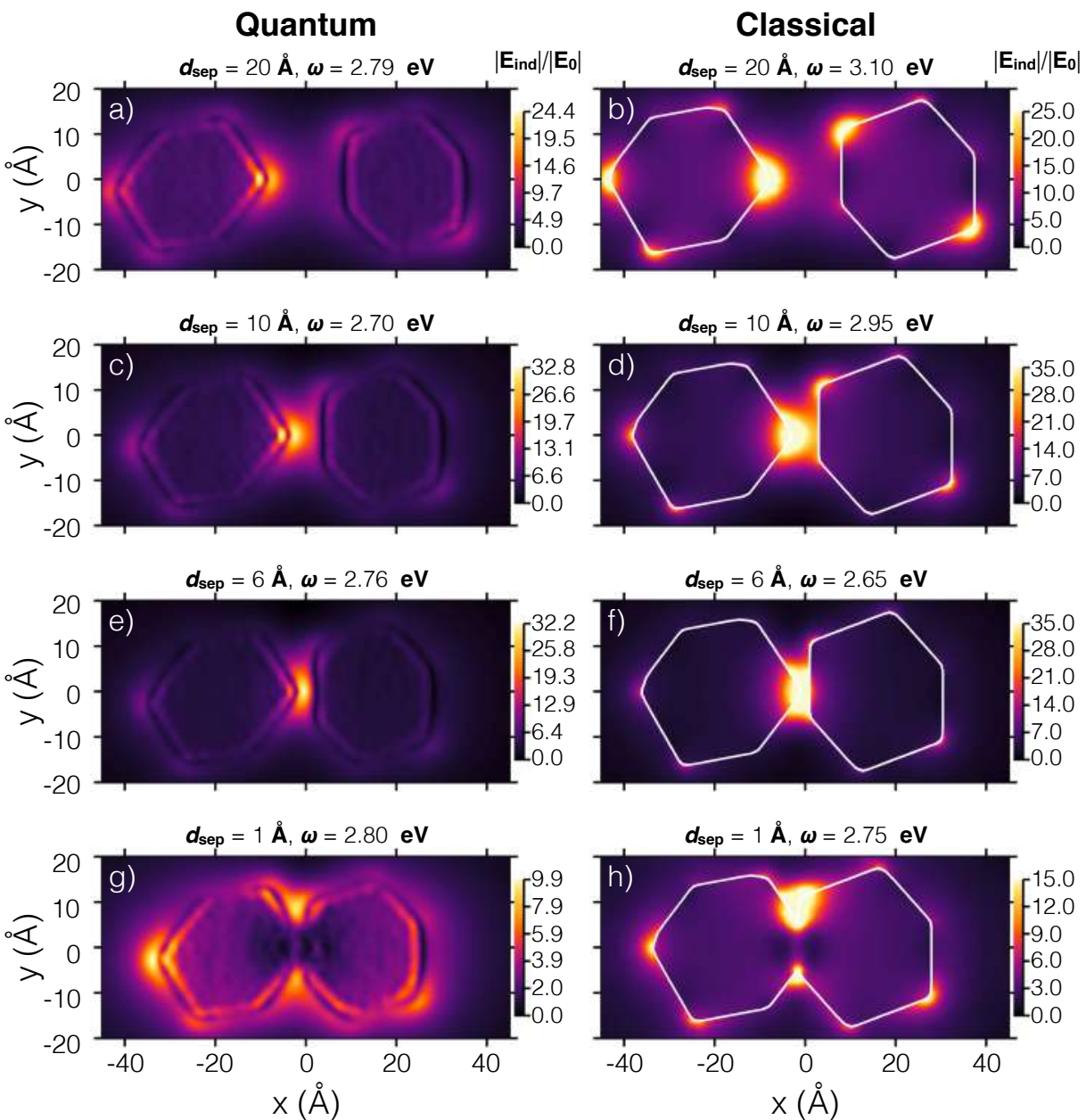

Figure 4: Induced near-field enhancement maps in the plane $(y, z)$ for a metallic dimer characterised by a tip-to-facet gap configuration [sketch of the geometry in Figure 3(b)] calculated with quantum TDDFT (left column) and with classical BEM (right column). Separation distances between particles are $d_{\text {sep }}=20 \AA$ panels $(\mathrm{a}, \mathrm{b}), d_{\text {sep }}=10 \AA$ panels $(\mathrm{c}, \mathrm{d})$, $d_{\text {sep }}=6 \AA$ panels $(\mathrm{e}, \mathrm{f})$ and $d_{\text {sep }}=1 \AA$ panels $(\mathrm{g}, \mathrm{h})$. The most intense resonances are selected for each separation distance. In most of the cases this resonance corresponds to the BDP, except those shown in panels (g,h), where the charge transfer CTP' is the most intense resonance.

effective mode volume gives a good description of the spatial localization of the particular mode, and can be obtained as an integration of the energy of the induced local fields of a mode, $\left|\mathbf{E}_{\text {ind }}(x, y, z)\right|^{2}$, normalised to the maximum local energy, $\left|\mathbf{E}_{\text {ind }}^{\max }\right|^{2}$, over the total volume, $V_{\text {tot }}$ :

$$
V_{\text {eff }}=\int \frac{\left|\mathbf{E}_{\text {ind }}(x, y, z)\right|^{2}}{\left|\mathbf{E}_{\text {ind }}^{\max }\right|^{2}} \mathrm{~d} V_{\text {tot }} .
$$

Thus, $V_{\text {eff }}$ provides the effective volume in which the field is localized in the middle of the gap. Figures $5(\mathrm{~d}-\mathrm{f})$ show the results as a function of $d_{\text {sep }}$ for the corresponding configurations and energies used in Figures 5(a-c). The dependence of $V_{\text {eff }}$ shows similar trends for all the configurations, with slight differences due to the details of each particular cavity. For $d_{\text {sep }}>5 \AA$, where the BDP mode dominates the response in all the gap configurations, there is a smooth increase of field confinement, i.e., decrease of $V_{\text {eff }}$, as the particles get closer, both in the quantum and classical descriptions. The classical description agrees very well with the quantum one: for a facet-to-facet configuration [Figure $5(\mathrm{~d})$ ] a confinement below $10 \mathrm{~nm}^{3}$ is possible limited by the extension of the atomic facets. An extreme situation is achieved with the presence of atomic-scale tips [Figures $5(\mathrm{e}, \mathrm{f})]$. In such a situation, the effective mode volumes reach subnanometric volumes, below $1 \mathrm{~nm}^{3}$. This level of confinement of light is the ultimate limit provided by condensed matter, and relies on the lightning rod effect associated to the potential profile driven by the electron density profile in the vicinity of a protruding atom. An important aspect is that the classical theory correctly describes this level of localization in spite of spill-out or dynamical screening effects, as demonstrated here.

For gap separation distances $d_{\text {sep }}<8 \AA$, the mode is quenched due to electron tunneling and thus the effective mode volume increases as the local field spreads out from the plasmonic cavity. This sets a threshold separation distance below which the classical description is 

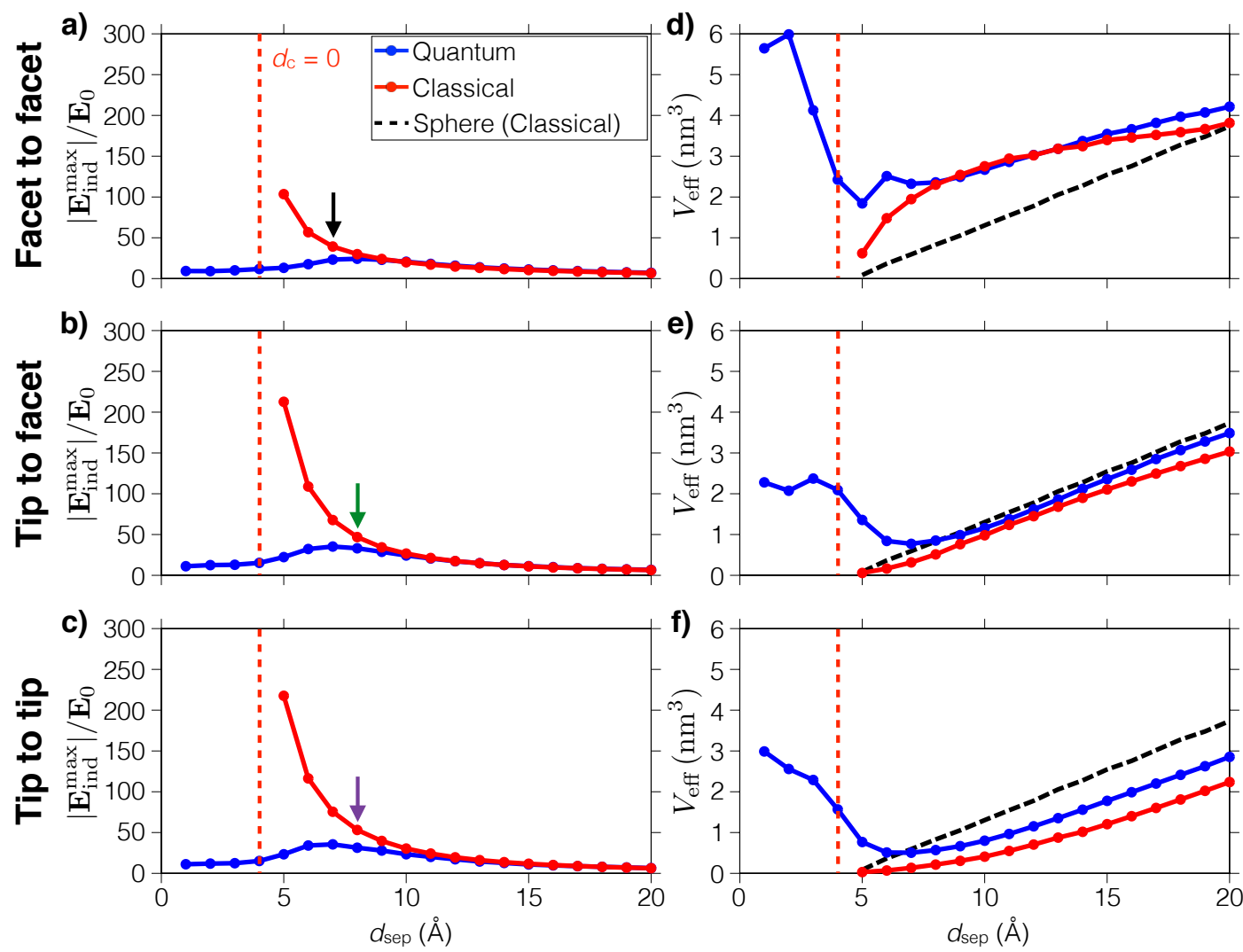

Figure 5: (a) - (c) Maximum local induced-field enhancement $\left|\mathbf{E}_{\text {ind }}^{\max }\right| / \mathbf{E}_{0}$ at the central plane bisecting the gap between two nanoparticles forming a plasmonic gap in the three configurations shown on the top of Figure 3: facet-to-facet, tip-to-facet and tip-to-tip, as a function of the separation distance between the particles, $d_{\text {sep }}$. The blue line stands for the TDDFT quantum calculations and the red line shows the classical BEM results. The vertical dashed red line marks the touching situation, $d_{c}=0$. Small arrows indicate the threshold separation distance below which the effect of quantum tunneling becomes noticeable. ${ }^{48}(\mathrm{~d})$ - (f) Effective mode volume $V_{\text {eff }}$ of the local field in the middle of the gap, as defined in the text (eq. 1), for all the three configurations. The data correspond to the energies for which the largest maximum of the induced near-field for each configuration and distance are obtained. The dashed black line addresses the effective mode volume corresponding to the gap of spherical particles of radius $r=1.85 \AA$.

not appropriate. This behavior depends much on the geometrical details of each particular configuration, as observed from the differences in the blue line of Figures $5(\mathrm{~d}-\mathrm{f})$.

\section{Conclusions}

The presence of atomic-scale features in metallic surfaces provides a mechanism of subnanometric localization of light. Unexpectedly, a lightning rod effect at the atomic scale is identified as responsible for this extra localization, induced by the electrical potential gradient produced by the electronic wavefunctions of the atomistic features. When superimposed on top of a nanometric plasmonic resonance, the lightning rod effect acts as a multiplier effect producing an extra factor of field enhancement which is characterized by a further spatial localization as compared to the supporting resonance, and a similar spectral distribution. The effective mode volumes of these atomistic features can reach subnano- metric dimensions thus taking the localization of light to the realm of picocavities which show mode volumes below $1 \mathrm{~nm}^{3}$. These results are fully validated by stateof-the-art quantum calculations of metallic nanostructures based on TDDFT which unambiguously show the existence of such picocavities. Our calculations show that a classical model within the solution of Maxwell's equations, where the atomic-scale features are described by sharp boundaries following the profile of the electron density associated with the atoms, is able to reproduce very satisfactorily the field localization and the effective mode volume in relevant canonical plasmonic nanoresonators such as in single metallic nanoparticles and in nanometric gaps formed by nanoparticle dimers. The current work has focused on Na nanoparticles, however the classical description of the atomic-scale lightning rod effect can be extended to other noble metals, such as $\mathrm{Ag}$ or $\mathrm{Au}$, widely used in nanoplasmonics, with the appropriate description of the dielectric function to account for interband trasitions in these materials. The validity of the classical approach to describe picocavi- 
ties, as demonstrated here, can be of practical importance to many experimental groups in surface-enhanced molecular spectroscopy, and in quantum nanophotonics, as the optical response of many large and complex plasmonic nanosystems can be successfully and appropriately modeled as outlined in this work. The existence of picocavities in metallic surfaces has probably been unconsciously revealed in many molecular spectroscopy experiments, enhanced by the action of surface plasmons, however, it is now, with the use of a simplified scheme as shown here, that the community can start to interpret the effects of atomic-scale features in spectroscopic signals in a compact and simple way by properly addressing the multiscale nature of the hosting cavities.

\section{Methods}

We perform atomistic, ab initio calculations of the optical response of the nanoparticles based on TDDFT. ${ }^{71}$ For the classical electromagnetic calculations, we implement the Boundary Element Method (BEM) ${ }^{72}$ within the framework of the local dielectric theory. We focus on icosahedral sodium clusters for such a comparison, although the method can be extended to other materials and shapes. In contrast to other BEM calculations, we take into account the finite curvature of the atomistic wedges and vertices using for this purpose the material atomic radius as a parameter. The details of these two approaches are given below:

i) Quantum calculation. Atomistic TDDFT : we apply our ab initio atomistic methods to obtain the optical response of single $\mathrm{Na}_{380}$ clusters, as well as dimers composed of two such clusters. The optical response of this system was already studied in Ref. ${ }^{45}$ with the same methodology, however, in the current results a more accurate description of the plasmonic resonance is achieved by improving several technical parameters, as detailed below and in the SI. $\mathrm{Na}_{380}$ is the largest cluster size for which the global minimum icosahedral symmetry (as described using an effective Murrell-Mottram potential ${ }^{73}$ ) is available. ${ }^{74}$ This structure ${ }^{75}$ was further relaxed using density functional theory (DFT), as implemented in the SIESTA code, ${ }^{76,77}$ within the generalized gradient approximation (GGA), with the use of the Perdew-Burke-Ernzerhof (PBE) functional. ${ }^{78}$ This choice improves the frequency of the plasmonic resonance with respect to that presented in Ref. ${ }^{45}$ due to a better description of the material's density. The geometry relaxation ensures the stability of the structure and that it corresponds at least to a local minimum of the DFT energy landscape of $\mathrm{Na}_{380}$. Subsequently, we obtained the TDDFT linear optical response of the cluster within the so-called adiabatic local density approximation (LDA) ${ }^{79,80}$ Using an iterative scheme developed by Koval and coworkers, ${ }^{71}$ we calculate the optical response at the TDDFT level for large systems at moderate computational cost. ${ }^{45,57,71,81}$

ii) Classical calculation. BEM: Maxwell's equations are solved within the local classical BEM approach. This model considers homogeneous dielectric functions to describe the response of the metal and the surrounding medium, which are assumed to be separated by abrupt boundaries. The calculations were carried out with the MNPBEM Toolbox. ${ }^{82,83}$ BEM requires the discretization of the boundary surfaces, instead of the whole volume of the different dielectric media.

For the size of the nanoparticles studied in this work, below the intrinsic mean free path of conduction electrons in bulk metals, surface scattering effects become important. In order to account for this effect in the classical approach, we include a correction to the freeelectron model (Drude model) following the prescriptions in the literature, ${ }^{84}$ with the assumption that surface scattering effects lead to a reduced effective mean free path $L_{\text {eff }}$, which adds a damping factor in the Drude dielectric function of the bulk metal. When specular reflection of electrons at the boundaries is assumed, as in the so-called Billiard model, ${ }^{85}$ the effective mean free path is given by $L_{\text {eff }}=4 V / A$, where $V$ and $A$ are the volume and area of the particle, respectively. ${ }^{86}$ Therefore, we adopted the Drude model of the dielectric function given by

$$
\varepsilon(\omega)=\epsilon_{\infty}-\frac{\omega_{p}^{2}}{\omega^{2}+i \omega\left(\gamma_{d}+\frac{\mathrm{vf}_{\mathrm{f}}}{L_{\mathrm{eff}}}\right)},
$$

where $\mathrm{v}_{\mathrm{f}}$ is the Fermi velocity $\left(\mathrm{v}_{\mathrm{f}}=1.07 \times 10^{6} \mathrm{~m} / \mathrm{s}\right.$ for $\mathrm{Na}$ ), and the intrinsic damping term is $\gamma_{d}=27.6$ meV. ${ }^{87}$ Other quantum effects such as nonlocality, spillout, or electron tunneling in close interparticle gaps are not considered in this dielectric function.

The main geometrical features (atomistic apexes, edges and facets) and shape of the particles considered in the atomistic TDDFT calculation are preserved in the BEM structure, which is modeled as a regular icosahedron with smoothened tips and edges [see Fig. 1(a,b)]. The radius of the circumscribing sphere of the regular icosahedron is $r=1.85 \mathrm{~nm}$, which is equivalent to half the distance between the atoms placed at the furthest tips of the atomistic structure, plus the Wigner-Seitz radius of sodium, in order to account for the size of the atoms. The tips and edges are further smoothened in such a way that their minimum curvature corresponds to the Wigner-Seitz radius of sodium $r_{\mathrm{Na}}=2.08 \AA$. Moreover, this procedure can be extrapolated for comparison with atomistic structures of different shapes and material. The differences between the smoothened and non-smoothened icosahedral particles of different sizes are discussed in the SI.

\section{Acknowledgement}

We would like to acknowledge fruitful discussions with J. J. Baumberg and V. Deckert. Financial support from Project No. FIS2016-80174-P and MAT2016-78293C6-4-R of MINECO, grant of Consolidated Groups at UPV/EHU (IT-756-13) of the Basque Government, and 
project 70NANB15H32 from U.S. Department of Commerce, National Institute of Standards and Technology, are acknowledged. M.U. acknowledges support from the University of the Basque Country through a PhD grant, as well as DIPC and CFM at the initial stages of this work. M.B. acknowledges support from the Departamento de Educación of the Basque Government through a PhD grant, as well as from Euskampus and the DIPC at the initial stages of this work. P.K. acknowledges financial support from the Fellows Gipuzkoa program of the Gipuzkoako Foru Aldundia through the FEDER funding scheme of the European Union, "Una manera de hacer Europa".

\section{Supporting Information Available}

The Supporting Information is available free of charge on the ACS Publications website.

Details of the ground-state calculations using SIESTA. Structure of the atomistic $\mathrm{Na}_{380}$ cluster and the plasmonic gaps used in the quantum TDDFT calculations and in the classical calculations within the Boundary Element Method (BEM). Influence of the edge rounding in the calculation of the optical response with BEM. Complementary atomistic calculations within classical Discrete Dipole Approximation (DDA). Polarization of the induced field in single nanoparticles. Derivation of the analytical expression of field enhancement in spheroidal bumps.

\section{References}

1. Kern, J.; Großmann, S.; Tarakina, N. V.; Häckel, T.; Emmerling, M.; Kamp, M.; Huang, J.S.; Biagioni, P.; Prangsma, J. C.; Hecht, B. AtomicScale Confinement of Resonant Optical Fields. Nano Lett. 2012, 12, 5504-5509.

2. Ward, D. R.; Huser, F.; Pauly, F.; Cuevas, J. C.; Natelson, D. Optical Rectification and Field Enhancement in a Plasmonic Nanogap. Nat. Nanotechnol. 2010, 5, 732-736.

3. Sigle, D. O.; Mertens, J.; Herrmann, L. O.; Bowman, R. W.; Ithurria, S.; Dubertret, B.; Shi, Y.; Yang, H. Y.; Tserkezis, C.; Aizpurua, J.; Baumberg, J. J. Monitoring Morphological Changes in 2D Monolayer Semiconductors Using Atom-Thick Plasmonic Nanocavities. ACS Nano 2015, 9, 825830.

4. Danckwerts, M.; Novotny, L. Optical Frequency Mixing at Coupled Gold Nanoparticles. Phys. Rev. Lett. 2007, 98, 026104.

5. Grillet, N.; Manchon, D.; Bertorelle, F.; Bonnet, C.; Broyer, M.; Cottancin, E.; Lermé, J.; Hillenkamp, M.; Pellarin, M. Plasmon Coupling in Silver Nanocube Dimers: Resonance Splitting Induced by Edge Rounding. ACS Nano 2011, 5, 9450-9462.
6. Moskovits, M. Surface-Enhanced Spectroscopy. Rev. Mod. Phys. 1985, 57, 783-826.

7. Nie, S.; Emory, S. R. Probing Single Molecules and Single Nanoparticles by Surface-Enhanced Raman Scattering. Science 1997, 275, 1102-1106.

8. Neubrech, F.; Pucci, A.; Cornelius, T. W.; Karim, S.; García-Etxarri, A.; Aizpurua, J. Resonant Plasmonic and Vibrational Coupling in a Tailored Nanoantenna for Infrared Detection. Phys. Rev. Lett. 2008, 101, 157403.

9. Ru, E. C. L.; Etchegoin, P. G. Single-Molecule Surface-Enhanced Raman Spectroscopy. Annu. Rev. Phys. Chem. 2012, 63, 65-87.

10. Xu, H.; Bjerneld, E. J.; Käll, M.; Börjesson, L. Spectroscopy of Single Hemoglobin Molecules by Surface Enhanced Raman Scattering. Phys. Rev. Lett. 1999, 83, 4357-4360.

11. Atwater, H. A.; Polman, A. Plasmonics for Improved Photovoltaic Devices. Nat. Mater. 2010, 9, 205-213.

12. Linic, S.; Christopher, P.; Ingram, D. B. PlasmonicMetal Nanostructures for Efficient Conversion of Solar to Chemical Energy. Nat. Mater. 2011, 10, 911-921.

13. Zia, R.; Schuller, J. A.; Chandran, A.; Brongersma, M. L. Plasmonics: The Next Chip-Scale Technology. Mater. Today 2006, 9, 20-27.

14. Akimov, A. V.; Mukherjee, A.; Yu, C. L.; Chang, D. E.; Zibrov, A. S.; Hemmer, P. R.; Park, H.; Lukin, M. D. Generation of Single Optical Plasmons in Metallic Nanowires Coupled to Quantum Dots. Nature 2007, 450, 402-406.

15. Zengin, G.; Wersäll, M.; Nilsson, S.; Antosiewicz, T. J.; Käll, M.; Shegai, T. Realizing Strong Light-Matter Interactions between SingleNanoparticle Plasmons and Molecular Excitons at Ambient Conditions. Phys. Rev. Lett. 2015, 114, 157401.

16. Santhosh, K.; Bitton, O.; Chuntonov, L.; Haran, G. Vacuum Rabi Splitting in a Plasmonic Cavity at the Single Quantum Emitter Limit. Nat. Commun. 2016, 11823.

17. Raschke, G.; Brogl, S.; Susha, A. S.; Rogach, A. L.; Klar, T. A.; ; Feldmann, J.; Fieres, B.; Petkov, N.; ; Bein, T.; Nichtl, A.; ; Kürzinger, K. Gold Nanoshells Improve Single Nanoparticle Molecular Sensors. Nano Lett. 2004, 4, 1853-1857. 
18. Anker, J. N.; Hall, W. P.; Lyandres, O.; Shah, N. C.; Zhao, J.; Van Duyne, R. P. Biosensing with Plasmonic Nanosensors. Nat Mater 2008, 7, 442-453.

19. Lal, S.; Clare, S. E.; Halas, N. J. Nanoshell-Enabled Photothermal Cancer Therapy: Impending Clinical Impact. Acc. Chem. Res. 2008, 41, 1842-1851.

20. Mühlschlegel, P.; Eisler, H.-J.; Martin, O. J. F.; Hecht, B.; Pohl, D. W. Resonant Optical Antennas. Science 2005, 308, 1607-1609.

21. Bharadwaj, P.; Deutsch, B.; Novotny, L. Optical Antennas. Adv. Opt. Photon. 2009, 1, 438-483.

22. Giannini, V.; Fernández-Domínguez, A. I.; Heck, S. C.; Maier, S. A. Plasmonic Nanoantennas: Fundamentals and Their Use in Controlling the Radiative Properties of Nanoemitters. Chem. Rev. 2011, 111, 3888-3912.

23. Biagioni, P.; Huang, J.-S.; Hecht, B. Nanoantennas for Visible and Infrared Radiation. Rep. Prog. Phys. 2012, 75, 024402.

24. Maier, S. A.; Kik, P. G.; Atwater, H. A.; Meltzer, S.; Harel, E.; Koel, B. E.; Requicha, A. A. G. Local Detection of Electromagnetic Energy Transport below the Diffraction Limit in Metal Nanoparticle Plasmon Waveguides. Nat Mater 2003, 2, 229-232.

25. Link, S.; El-Sayed, M. A. Spectral Properties and Relaxation Dynamics of Surface Plasmon Electronic Oscillations in Gold and Silver Nanodots and Nanorods. J. Phys. Chem. B 1999, 103, 8410-8426.

26. Jin, R.; Cao, Y.; Mirkin, C. A.; Kelly, K. L.; Schatz, G. C.; Zheng, J. G. Photoinduced Conversion of Silver Nanospheres to Nanoprisms. Science 2001, 294, 1901-1903.

27. Averitt, R. D.; Sarkar, D.; Halas, N. J. Plasmon Resonance Shifts of Au-Coated $\mathrm{Au}_{2} \mathrm{~S}$ Nanoshells: Insight into Multicomponent Nanoparticle Growth. Phys. Rev. Lett. 1997, 78, 4217-4220.

28. Recio, F. J.; Zabala, N.; Rivacoba, A.; Crespo, P.; Ayuela, A.; Echenique, P. M.; Hernando, A. Optical Resonances of Colloidal Gold Nanorods: From Seeds to Chemically Thiolated Long Nanorods. J. Phys. Chem. C 2015, 119, 7856-7864.

29. Myroshnychenko, V.; Rodríguez-Fernandez, J.; Pastoriza-Santos, I.; Funston, A. M.; Novo, C.; Mulvaney, P.; Liz-Marzán, L. M.; García de Abajo, F. J. Modelling the Optical Response of Gold Nanoparticles. Chem. Soc. Rev. 2008, 37, 1792-1805.
30. Aizpurua, J.; Hanarp, P.; Sutherland, D. S.; Käll, M.; Bryant, G. W.; García de Abajo, F. J. Optical Properties of Gold Nanorings. Phys. Rev. Lett. 2003, 90, 057401.

31. Aizpurua, J.; Bryant, G. W.; Richter, L. J.; García de Abajo, F. J.; Kelley, B. K.; Mallouk, T. Optical Properties of Coupled Metallic Nanorods for Field-Enhanced Spectroscopy. Phys. Rev. B 2005, 71, 235420.

32. Funston, A. M.; Novo, C.; Davis, T. J.; Mulvaney, P. Plasmon Coupling of Gold Nanorods at Short Distances and in Different Geometries. Nano Lett. 2009, 9, 1651-1658.

33. Hao, F.; Nehl, C. L.; Hafner, J. H.; Nordlander, P. Plasmon Resonances of a Gold Nanostar. Nano Lett. 2007, 7, 729-732.

34. Novikov, S. M.; Sánchez-Iglesias, A.; Schmidt, M. K.; Chuvilin, A.; Aizpurua, J.; Grzelczak, M.; Liz-Marzán, L. M. Gold Spiky Nanodumbbells: Anisotropy in Gold Nanostars. Part. Part. Syst. Charact. 2014, 31, 77-80.

35. Pérez-González, O.; Zabala, N.; Borisov, A. G.; Halas, N. J.; Nordlander, P.; Aizpurua, J. Optical Spectroscopy of Conductive Junctions in Plasmonic Cavities. Nano Lett. 2010, 10, 3090-3095.

36. Townsend, E.; Bryant, G. W. Plasmonic Properties of Metallic Nanoparticles: The Effects of Size Quantization. Nano Lett. 2012, 12, 429-434.

37. Scholl, J. A.; Koh, A. L.; Dionne, J. A. Quantum Plasmon Resonances of Individual Metallic Nanoparticles. Nature 2012, 483, 421-427.

38. Monreal, R. C.; Antosiewicz, T. J.; Apell, S. P. Competition between Surface Screening and Size Quantization for Surface Plasmons in Nanoparticles. New J. Phys. 2013, 15, 083044.

39. Negre, C. F. A.; Perassi, E. M.; Coronado, E. A.; Sánchez, C. G. Quantum Dynamical Simulations of Local Field Enhancement in Metal Nanoparticles. J. Phys.: Condens. Matter 2013, 25, 125304.

40. Iida, K.; Noda, M.; Ishimura, K.; Nobusada, K. First-Principles Computational Visualization of Localized Surface Plasmon Resonance in Gold Nanoclusters. J. Phys. Chem. A 2014, 118, 1131711322 .

41. Feibelman, P. J. Microscopic Calculation of Electromagnetic Fields in Refraction at a Jellium-Vacuum Interface. Phys. Rev. B 1975, 12, 1319-1336. 
42. Liebsch, A.; Schaich, W. L. Influence of a Polarizable Medium on the Nonlocal Optical Response of a Metal Surface. Phys. Rev. B 1995, 52, 1421914234 .

43. Teperik, T. V.; Nordlander, P.; Aizpurua, J.; Borisov, A. G. Robust Subnanometric Plasmon Ruler by Rescaling of the Nonlocal Optical Response. Phys. Rev. Lett. 2013, 110, 263901.

44. Zhang, P.; Feist, J.; Rubio, A.; García-González, P.; García-Vidal, F. J. Ab initio Nanoplasmonics: The Impact of Atomic Structure. Phys. Rev. B 2014, 90, 161407.

45. Barbry, M.; Koval, P.; Marchesin, F.; Esteban, R.; Borisov, A. G.; Aizpurua, J.; Sánchez-Portal, D. Atomistic Near-Field Nanoplasmonics: Reaching Atomic-Scale Resolution in Nanooptics. Nano Lett. 2015, 15, 3410-3419.

46. Zuloaga, J.; Prodan, E.; Nordlander, P. Quantum Description of the Plasmon Resonances of a Nanoparticle Dimer. Nano Lett. 2009, 9, 887-891.

47. Marinica, D.; Kazansky, A.; Nordlander, P.; Aizpurua, J.; Borisov, A. G. Quantum Plasmonics: Nonlinear Effects in the Field Enhancement of a Plasmonic Nanoparticle Dimer. Nano Lett. 2012, 12, 1333-1339.

48. Esteban, R.; Borisov, A. G.; Nordlander, P.; Aizpurua, J. Bridging Quantum and Classical Plasmonics with a Quantum-Corrected Model. Nat. Commun. 2012, 3, 825 .

49. Ciracì, C.; Pendry, J. B.; Smith, D. R. Hydrodynamic Model for Plasmonics: A Macroscopic Approach to a Microscopic Problem. ChemPhysChem 2013, 14, 1109-1116.

50. Mortensen, N. A.; Raza, S.; Wubs, M.; Søndergaard, T.; Bozhevolnyi, S. I. A Generalized Non-Local Optical Response Theory for Plasmonic Nanostructures. Nat. Commun. 2014, 5, 3809 .

51. Esteban, R.; Zugarramurdi, A.; Zhang, P.; Nordlander, P.; Garcia-Vidal, F. J.; Borisov, A. G.; Aizpurua, J. A Classical Treatment of Optical Tunneling in Plasmonic Gaps: Extending the Quantum Corrected Model to Practical Situations. Faraday Discuss. 2015, 178, 151-183.

52. Jensen, L.; Autschbach, J.; Schatz, G. C. Finite Lifetime Effects on the Polarizability within TimeDependent Density-Functional Theory. J. Chem. Phys. 2005, 224115.
53. Zhao, L.; Jensen, L.; Schatz, G. C. PyridineAg20 Cluster: A Model System for Studying SurfaceEnhanced Raman Scattering. J. Am. Chem. Soc. 2006, 128, 2911-2919.

54. Aikens, C. M.; Li, S.; Schatz, G. C. From Discrete Electronic States to Plasmons: TDDFT Optical Absorption Properties of $\mathrm{Ag} \mathrm{n}(\mathrm{n}=10,20,35$, 56, 84, 120) Tetrahedral Clusters. J. Phys. Chem. C 2008, 112, 11272-11279.

55. Malola, S.; Lehtovaara, L.; Enkovaara, J.; Hkkinen, H. Birth of the Localized Surface Plasmon Resonance in Monolayer-Protected Gold Nanoclusters. ACS Nano 2013, 7, 10263-10270.

56. Kuisma, M.; Sakko, A.; Rossi, T. P.; Larsen, A. H.; Enkovaara, J.; Lehtovaara, L.; Rantala, T. T. Localized Surface Plasmon Resonance in Silver Nanoparticles: Atomistic First-Principles TimeDependent Density-Functional Theory Calculations. Phys. Rev. B 2015, 91, 115431.

57. Marchesin, F.; Koval, P.; Barbry, M.; Aizpurua, J.; Sánchez-Portal, D. Plasmonic Response of Metallic Nanojunctions Driven by Single Atom Motion: Quantum Transport Revealed in Optics. ACS Photonics 2016, 3, 269-277.

58. Runge, E.; Gross, E. K. U. Density-Functional Theory for Time-Dependent Systems. Phys. Rev. Lett. 1984, 52, 997-1000.

59. Parr, R. G.; Yang, W. Density-Functional Theory of Atoms and Molecules; Oxford university press, 1989; Vol. 16.

60. Zhang, Y.; Meng, Q.-S.; Zhang, L.; Luo, y.; Yu, Y.J.; Zhang, Y.; Esteban, R.; Aizpurua, J.; Luo, Y.; Yang, J.-L.; Dong, Z.-C.; Hou, J. Sub-Nanometre Control of the Coherent Interaction between a Single Molecule and a Plasmonic Nanocavity. Nat. Commun. 2017, 15225 .

61. Benz, F.; Schmidt, M. K.; Dreismann, A.; Chikkaraddy, R.; Zhang, Y.; Demetriadou, A.; Carnegie, C.; Ohadi, H.; de Nijs, B.; Esteban, R.; Aizpurua, J.; Baumberg, J. J. Single-Molecule Optomechanics in "picocavities". Science 2016, 354, 726-729.

62. Trautmann, S.; Aizpurua, J.; Götz, I.; Undisz, A.; Dellith, J.; Schneidewind, H.; Rettenmayr, M.; Deckert, V. A Classical Description of Subnanometer Resolution by Atomic Features in Metallic Structures. Nanoscale 2017, 391-401.

63. Alabastri, A.; Yang, X.; Manjavacas, A.; Everitt, H. O.; Nordlander, P. Extraordinary Light-Induced Local Angular Momentum near Metallic Nanoparticles. ACS Nano 2016, 10, $4835-4846$. 
64. Romero, I.; Aizpurua, J.; Bryant, G. W.; de Abajo, F. J. G. Plasmons in Nearly Touching Metallic Nanoparticles: Singular Response in the Limit of Touching Dimers. Opt. Express 2006, 14, 9988-9999.

65. Prodan, E.; Radloff, C.; Halas, N. J.; Nordlander, P. A Hybridization Model for the Plasmon Response of Complex Nanostructures. Science 2003 302, 419-422.

66. Haran, G. Single-Molecule Raman Spectroscopy: A Probe of Surface Dynamics and Plasmonic Fields. Acc. Chem. Res. 2010, 43, 1135-1143.

67. Ward, D. R.; Grady, N. K.; Levin, C. S.; Halas, N. J.; Wu, Y.; Nordlander, P.; Natelson, D. Electromigrated Nanoscale Gaps for Surface-Enhanced Raman Spectroscopy. Nano Lett. 2007, 7, 1396-1400.

68. Willets, K. A.; Duyne, R. P. V. Localized Surface Plasmon Resonance Spectroscopy and Sensing. Annu. Rev. Phys. Chem. 2007, 58, 267-297.

69. Lim, D.-K.; Jeon, K.-S.; Kim, H. M.; Nam, J.-M.; Suh, Y. D. Nanogap-Engineerable Raman-Active Nanodumbbells for Single-Molecule Detection. Nat Mater 2010, 9, 60-67.

70. Esteban, R.; Borisov, A. G.; Nordlander, P.; Aizpurua, J. Bridging Quantum and Classical Plasmonics with a Quantum-Corrected Model. Nat.Commun. 2012, 3, 825 .

71. Koval, P.; Marchesin, F.; Foerster, D.; SánchezPortal, D. Optical Response of Silver Clusters and Their Hollow Shells from Linear-Response TDDFT. J. Phys.: Condens. Matter 2016, 28, 214001.

72. García de Abajo, F. J.; Howie, A. Retarded Field Calculation of Electron Energy Loss in Inhomogeneous Dielectrics. Phys. Rev. B 2002, 65, 115418.

73. Murrell, J. N.; Mottram, R. E. Potential Energy Functions for Atomic Solids. Mol. Phys. 1990, 69, $571-585$.

74. Noya, E. G.; Doye, J. P.; Wales, D. J.; Aguado, A. Geometric Magic Numbers of Sodium Clusters: Interpretation of the Melting Behaviour. Eur. Phys. J. D 2007, 43, 57-60.

75. The initial structure of the Na380 cluster was downloaded from the Cambridge Cluster Database. Wales, D. J.; Doye, J. P. K.; Dullweber, A.; Hodges, M.; Naumkin, F.; Calvo, F.; Hernańdez-Rojas, J.; Middleton, T. F. http:// www-wales.ch.cam.ac.uk/CCD.html.
76. Sánchez-Portal, D.; Ordejón, P.; Artacho, E.; Soler, J. M. Density-Functional Method for Very Large Systems with LCAO Basis Sets. Int. J. Quantum Chem. 1997, 65, 453-461.

77. Soler, J. M.; Artacho, E.; Gale, J. D.; García, A.; Junquera, J.; Ordejón, P.; Sánchez-Portal, D. The SIESTA Method for Ab Initio Order-N Materials Simulation. J. Phys. Condens. Matter 2002, 14, 2745 .

78. Perdew, J. P.; Burke, K.; Ernzerhof, M. Generalized Gradient Approximation Made Simple. Phys. Rev. Lett. 1996, 77, 3865-3868.

79. Kohn, W.; Sham, L. J. Self-Consistent Equations Including Exchange and Correlation Effects. Phys. Rev. 1965, 140, A1133-A1138.

80. Perdew, J. P.; Zunger, A. Self-Interaction Correction to Density-Functional Approximations for Many-Electron Systems. Phys. Rev. B 1981, 23, 5048-5079.

81. Manjavacas, A.; Marchesin, F.; Thongrattanasiri, S.; Koval, P.; Nordlander, P.; SánchezPortal, D.; de Abajo, F. J. G. Tunable Molecular Plasmons in Polycyclic Aromatic Hydrocarbons. ACS Nano 2013, 7, 3635-3643.

82. Hohenester, U.; Trügler, A. MNPBEM - a Matlab Toolbox for the Simulation of Plasmonic Nanoparticles. Comput. Phys. 2012, 183, 370 - 381.

83. Hohenester, U. Simulating Electron Energy Loss Spectroscopy with the MNPBEM Toolbox. Comput. Phys. 2014, 185, $1177-1187$.

84. Moroz, A. Electron Mean Free Path in a Spherical Shell Geometry. J. Phys. Chem. C 2008, 112, 10641-10652.

85. Kreibig, U.; Fragstein, C. The Limitation of Electron Mean Free Path in Small Silver Particles. $Z$. Phys. 1969, 224, 307-323.

86. Chernov, N. Lyapunov Instability of the BoundaryDriven Chernov-Lebowitz Model for Stationary Shear Flow. J. Stat. Phys. 1997, 88, 825-842.

87. Kittel, C. Introduction to Solid State Physics, 7th ed.; John Wiley and Sons, 1996. 


\section{Graphical TOC Entry}

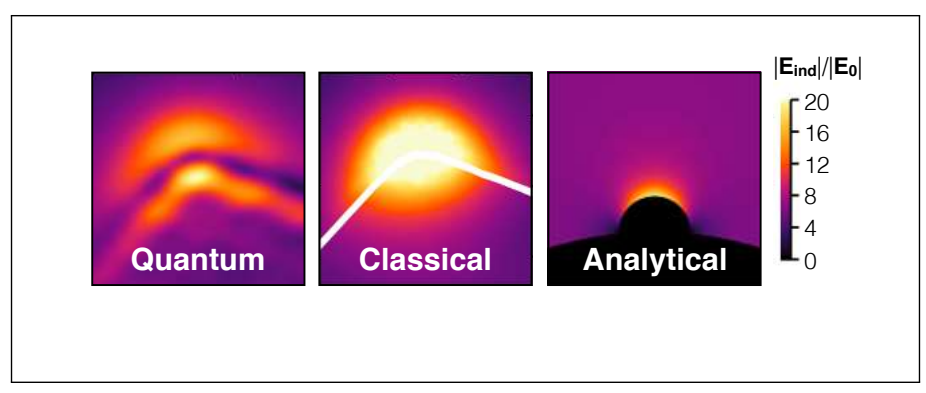




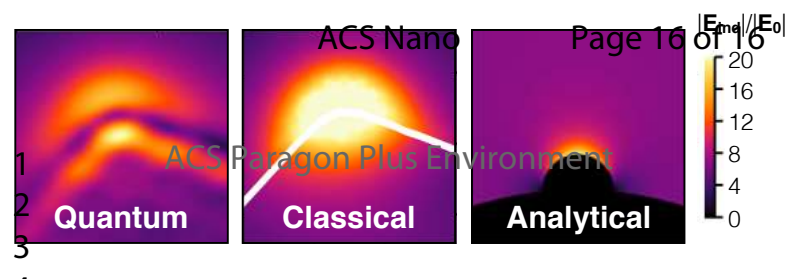

